•综述・

\title{
大蜜蜂的生物学特性、面临威胁与保护策略
}

\author{
杨 培 ${ }^{1}$ 彭艳琼 $^{*}$ 赵荣华 ${ }^{2}$ 杨大荣 ${ }^{1}$ \\ 1 (中国科学院西双版纳热带植物园热带森林生态学重点实验室, 云南预腊 666303) \\ 2 (云南中医学院, 昆明 650500)
}

\begin{abstract}
摘要: 昆虫传粉在维持植物的有性繁殖、物种形成及生态系统稳定中扮演着重要角色, 而野生传粉昆虫为生态系 统提供了巨大的传粉服务功能。大蜜蜂(Apis dorsata) 为亚洲特有的一种野生传粉昆虫, 是热带地区多种植物和农 作物的有效传粉者, 在保障热带生物多样性及作物产量中有不可或缺的作用。但受全球气候变化、人类活动和生 境恶化等因素的影响, 其种群数量日益减少, 开展大蜜蜂种质资源保护势在必行。本文综述了大蜜蜂筑巢、迁飞 和传粉服务功能, 分析了人为猎取蜂巢, 栖息生境遭受破坏, 杀虫剂和除草剂滥用, 昆虫、螨类和病原物侵染, 气 候变化等威胁种群的因素, 以期从强化大蜜蜂基础研究和保护、推动生态农业发展、建立适合大蜜蜂迁飞生态廊 道、加强检验检疫及科学合理利用大蜜蜂种质资源等方面制定相应的保护措施。
\end{abstract}

关键词: 大蜜蜂; 野生传粉昆虫; 传粉服务; 威胁因素; 保护

\section{Biological characteristics, threat factors and conservation strategies for the giant honey bee Apis dorsata}

\author{
Pei Yang ${ }^{1}$, Yanqiong Peng ${ }^{1 *}$, Ronghua Zhao ${ }^{2}$, Darong Yang ${ }^{1}$ \\ 1 Key Laboratory of Tropical Forest Ecology, Xishuangbanna Tropical Botanical Garden, Chinese Academy of Sciences, \\ Mengla, Yunnan 666303 \\ 2 Yunnan University of Traditional Chinese Medicine, Kunming 650500
}

\begin{abstract}
Insect pollinators play a vital role in plant sexual reproduction. Pollinators facilitate cross-pollination that in turn promotes genetic diversity, mediates plant speciation, and contributes to ecosystem stability. However, the abundance, diversity and health of wild pollinators are threatened by human activities such as anthropogenic climate impacts, habitat destruction and environmental pollutants, and the impact of these human activities on ecosystems is likely to increase. Despite recognizing the importance of wild pollinators and the implementation of targeted conservation programs, the contemporary threats of wild insect pollinators remain poorly understood. For the giant honey bee (Apis dorsata), an important wild pollinator and honey producer in tropical rainforests and agricultural areas across Asia, here we describe nest characteristics, colony migration and pollination role and review threats to their conservation. We found that A. dorsata nests featured a single honeycomb hanging from the branches of large trees. The bees undertook long distance migrations to locate seasonally ephemeral forage sources but regularly returned to previous nesting sites. We identified several anthropogenic activities that posed significant threats to A. dorsata conservation: harvesting entire colonies, deforestation, pesticide and herbicide utilization, parastioids, mites, pathogens and climate change. Based on our study, we recommend several conservation initiatives to promote wild $A$. dorsata populations, which include artificial domestication, developing ecological agriculture, establishing of ecological corridors, inspection and quarantine controls on domesticated colonies, and sustainable utilization of the floral resources used by A. dorsata. We hope that this review will stimulate future research on giant honey bees whilst playing a significant role in their conservation and sustainable utilization.
\end{abstract}

Key words: Apis dorsata; wild insect pollinators; pollination services; threat factors; conservation

收稿日期: 2018-02-05; 接受日期: 2018-03-28

基金项目: 国家重点研发计划(2017YFC1700704)、南药研究协同创新中心基金资助项目(30270100500)和中国科学院“一三五”专项(2017XTBG-F01)

* 通讯作者 Author for correspondence. E-mail: pengyq@xtbg.ac.cn 
昆虫传粉协助植物异花授粉, 有利于维持植物 的遗传多样性, 增强后代变异性和生境适应性, 推 动物种进化, 在维持生物多样性和生态系统稳定及 食物安全方面具有不可替代的作用(Wardhaugh, 2015; Ollerton, 2017)。根据生物多样性与生态系统 服务政府间科学-政策平台(Intergovernmental Science-Policy Platform on Biodiversity and Ecosystem Services, IPBES)发布的“传粉者、传粉和粮食生产” 评估报告, 动物传粉每年创造的经济价值高达 2,350-5,770亿美元(Potts et al, 2016), 而昆虫传粉在 整个服务链条中占比近 $90 \%$ (Wardhaugh, 2015)。目 前已知传粉昆虫有 35 万多种, 分为野生和家养两类: 野生传粉昆虫包括蜂类、蝇类、蝶类、蛾类、甲虫、 蓟马等, 贡献了约70\%的传粉服务; 家养传粉昆虫 以西方蜜蜂(Apis mellifera)和东方蜜蜂(A. cerana)为 主, 在生态系统中提供了 $30 \%$ 的传粉服务(Alexandra-Maria et al, 2007; Breeze et al, 2014; Wardhaugh, 2015)。然而, 近年来由于全球气候变化、人 为活动加剧、生态环境恶化等因素的影响, 传粉昆 虫的种类和数量日益减少(Potts et al, 2010; Lebuhn et al, 2013; Ghazoul, 2015), 特别是野生蜜蜂(包括 蜜蜂科、切叶蜂科、准蜂科等蜜蜂总科昆虫)减少更
为突出(Pauw, 2007; Cameron et al, 2011; Burkle et al, 2013; Ollerton et al, 2014)。据统计，全球有16.5\%的 传粉昆虫受到威胁，约 $40 \%$ 的访花蜜蜂和蝴蝶面临 灭绝，引起407个植物-传粉者互作网络关系缺失, 其中 $45 \%$ 的传粉网络丧失是因野生蜜蜂种类的灭绝 引起的(Burkle et al, 2013; Ollerton, 2017), 野生传 粉蜜蜂的减少和消失正在也必将对生态环境和人 类造成重大的影响。

大蜜蜂(Apis dorsata)隶属于膜翅目蜜蜂科蜜蜂 属(Apis), 为亚洲特有的一种野生蜜蜂, 因其个体 较蜜蜂属其他种类大，故称为大蜜蜂，又因其蜂巢 单脾成排，俗称排蜂，主要分布于我国的云南、广 西和海南，以及东南亚的泰国、缅甸、柬埔寨、越 南、新加坡、老挝和印度尼西亚，部分延伸至巴基 斯坦、印度和尼泊尔等地(图1), 属于东洋界区系。 (吴燕如, 2000; Hepburn \& Radloff, 2011; Shankar et al, 2017)。大蜜蜂是热带雨林林冠层植物和多种 热带农作物的有效传粉昆虫，有很好的传粉服务功 能(张正松, 1974; Momose et al, 1998; Raghunandan \& Basavarajappa, 2014)。但由于大蜜蜂蜂巢大, 产 蜜量高, 巢内蜂蜡多, 具有丰富的营养价值和较高 的经济价值, 致使采蜜人大肆猎取蜂巢(张正松,

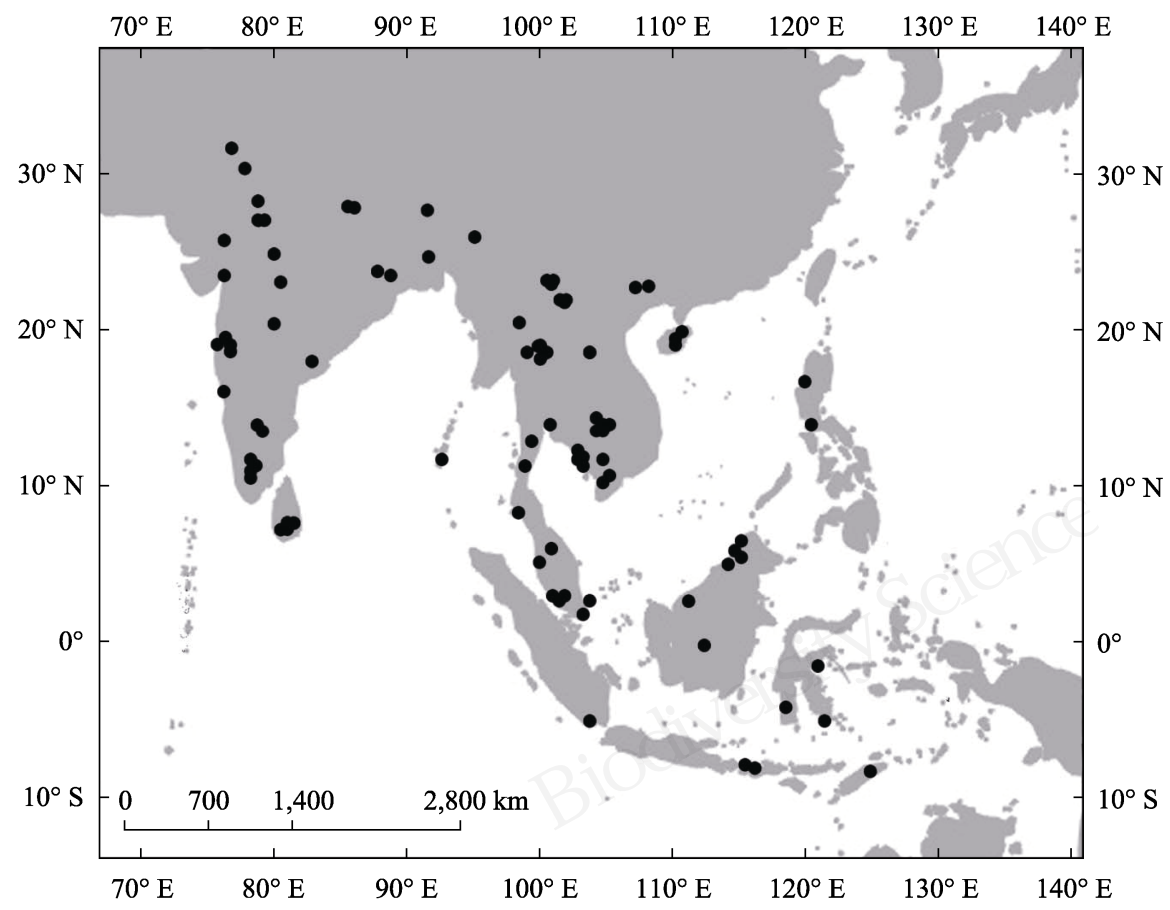

图1 大蜜蜂的地理分布图(黑点表示文献记录的大蜜蜂的分布区)

Fig. 1 The geographical distribution of Apis dorsata. The black dots show the distribution sites in references. 
1974; Lahjie \& Seibert, 1990; Soman \& Kshirsagar, 1991), 同时受森林滥伐以及城镇化加速等人为因 素的影响, 大蜜蜂种群数量锐减, 有些地方甚至达 濒危程度(Nath et al, 1994)。因此, 有必要对大蜜蜂 的筑巢、迁飞和传粉服务功能进行总结, 结合全球 气候变化, 分析探讨其种群所面临的潜在威胁, 提 出对其种质资源保护的对策, 以期提高大蜜蜂的传 粉服务功能并保障其资源的可持续利用。

\section{大蜜蜂的生物学特性}

\section{1 筑巢与迁飞行为}

大蜜蜂的筑巢与迁飞行为和蜜粉源植物的分 布、花期有着密切关系, 而蜜粉源植物的物候又决 定了大蜜蜂的巢址选择、迁飞时间及路线(Itioka et al, 2001; Woyke et al, 2012)。大蜜蜂偏爱在空旷干净 的空间筑巢, 如原始森林中的高大乔木、悬崖及建 筑物上(图2)。同树或建筑物上可筑20余巢，最多可 达200个, 巢间距最小的仅有几厘米; 单巢直径可 达200 cm, 高70 cm (Paar et al, 2004; Oldroyd \& Nanork, 2009; Rattanawannee et al, 2013)。大蜜蜂在 寻找筑巢树时，选择植物种类没有明显的偏好性， 目前已发现可在 18 科 50 种高大乔木上筑巢，常见于 木棉科木棉 (Bombax ceiba)、楝科紫椿 (Toona sureni)、桑科聚果榕(Ficus racemosa) 和高山榕(F. altissima)等树木的结实枝干上，枝干距地面 $10 \mathrm{~m}$ 以 上, 且与地面夹角小于 $60^{\circ}$, 与朝向无关, 但 $90 \%$ 以 上的巢穴均有树冠的遮蔽, 可避免鸟类、大黄蜂等 天敌的直接捕食(Starr et al, 1987; Nagir et al, 2016; Sihag, 2017)。

大蜜蜂是典型的热带迁飞性昆虫, 一年至少迁 飞两次, 迁飞距离可达200 km (Paar et al, 2000)。迁 飞条件主要取决于可利用的食物资源(Dyer \& Seeley, 1994; Paar et al, 2000; Woyke et al, 2012), 并与 当地气候、幼虫和蛹被寄生的情况、生境破坏等因 素有关(张正松, 1974; Paar et al, 2004; 曹联飞和胡 福良, 2012; Makinson et al, 2014)。例如, 尼泊尔地 区的部分植物在 12 月上旬进入始花期, 此时大蜜蜂 开始迁入, 至翌年4-5月花期结束, 大蜜蜂逐渐离 开; 而巴基斯坦地区的大蜜蜂出现在3-5月，印度 尼西亚6-7月最多(Woyke et al, 2012)。同时, 大蜜蜂 常沿江河路线迁徙，并根据花期即食物资源选择离 水源较近的地方, 尤其在周围有高大乔木存在且处
于盛花期的果园临时聚集休整, 如芒果园, 大蜂群 可分解为几个小巢, 花期结束后大蜜蜂即刻集体离 巢继续迁飞(Robinson, 2012)。与其他蜜蜂不同，大 蜜蜂迁回时若旧巢未被破坏, 它们仍会利用旧巢重 建新巢, 有些甚至在离开两年后仍能够再次回到旧 巢(Neumann et al, 2000; Oldroyd et al, 2000)。但是, 关于大蜜蜂如何在长时间离开后, 特别是先前随蜂 王离开的工蜂已经不复存在, 时空记忆信息(Moreyra et al, 2017)也已消退, 后代如何重新找到旧巢 的机制并不清楚，仅有一些推测认为这与蜂群的组 织结构及其内部信息调控有关(Woyke et al, 2012)。

\section{2 大蜜蜂的传粉特性}

热带雨林林冠层乔木及其附生植物通常距地 面50 m以上，植株间隔远，且这些植物花期较短, 时间集中，无规律，如一些龙脑香科植物2-10年才 开一次花, 但开花特性与大蜜蜂的传粉生态学特征 恰好匹配。大蜜蜂迁飞和分巢时，其种群数量可在 短时间内大规模增加，可以满足雨林林冠层植物集 中开花传粉的需要(Momose et al, 1998; Oldroyd \& Nanork, 2009; Somanathan et al, 2009)。例如，盛花 期时, 雨林中大蜜蜂蜂巢数量会明显增加, 而花少 时蜂巢也较少, 说明食物资源充足时能够吸引数量 众多的大蜜蜂到来。同时，大蜜蜂的昼夜活动节律 较广, 不仅在光线充足的白天活动, 还可在光线暗 淡的黎明和傍晚活动(Dyer, 1985; Somanathan et al, 2009), 这是其他社会性传粉昆虫所不具备的。而这 种生活习性可满足一些在该时段开花植物的传粉 需求, 如龙脑香科的Dipterocarpus tempehes和五桠 果科的Dillenia excels凌晨5:00开花，其他龙脑香属 (Dipterocarpus) 的一些植物在傍晚 6:00 开花(Momose et al, 1998), 睡莲科白睡莲(Nymphaea alba)于 夜间 9:00至清晨 6:00开花，均是依靠大蜜蜂完成传 粉。此外, 大蜜蜂有较强的飞行能力, 可有效突破 林冠层植株间距较大的限制, 因而被认为是在雨林 生境碎片化加重的情况下，实现花粉散布和基因流 动的主要动力(Noreen et al, 2016)。

由于对大蜜蜂传粉生物学研究较少, 罕有关于 其为林下植物传粉的报道, 然而, 我们观察到它们 是多种热带植物的主要传粉昆虫(图3)。此外，尽管 大蜜蜂可以泛化地为多种植物传粉, 但一些植物却 专性地依赖其传粉实现有性繁殖。金虎尾科风筝果 (Hiptage benghalensis)是一种亚洲特有植物，其花 

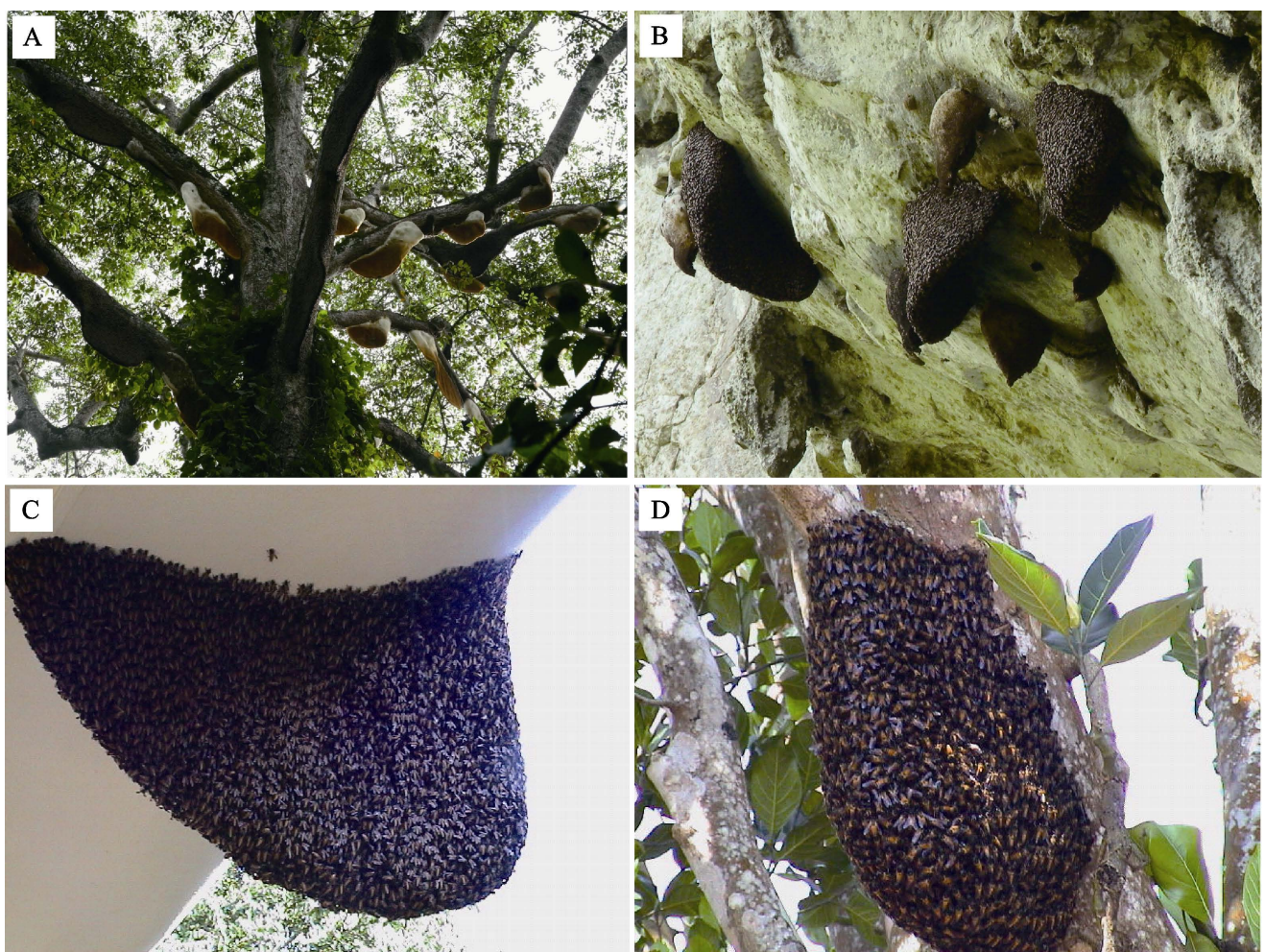

图2 大蜜蜂筑巢地。A：木棉枝干的蜂巢; B: 悬崖上的蜂巢; C: 屋檐下的蜂巢; D: 工蜂聚集状

Fig. 2 Colony sites of Apis dorsata. A-B, Separate colonies aggregated on the branches of Bombax ceiba (A) and cliff (B); C, The colony under the eave; D, Multiple-layer curtain formed by A. dorsata workers.
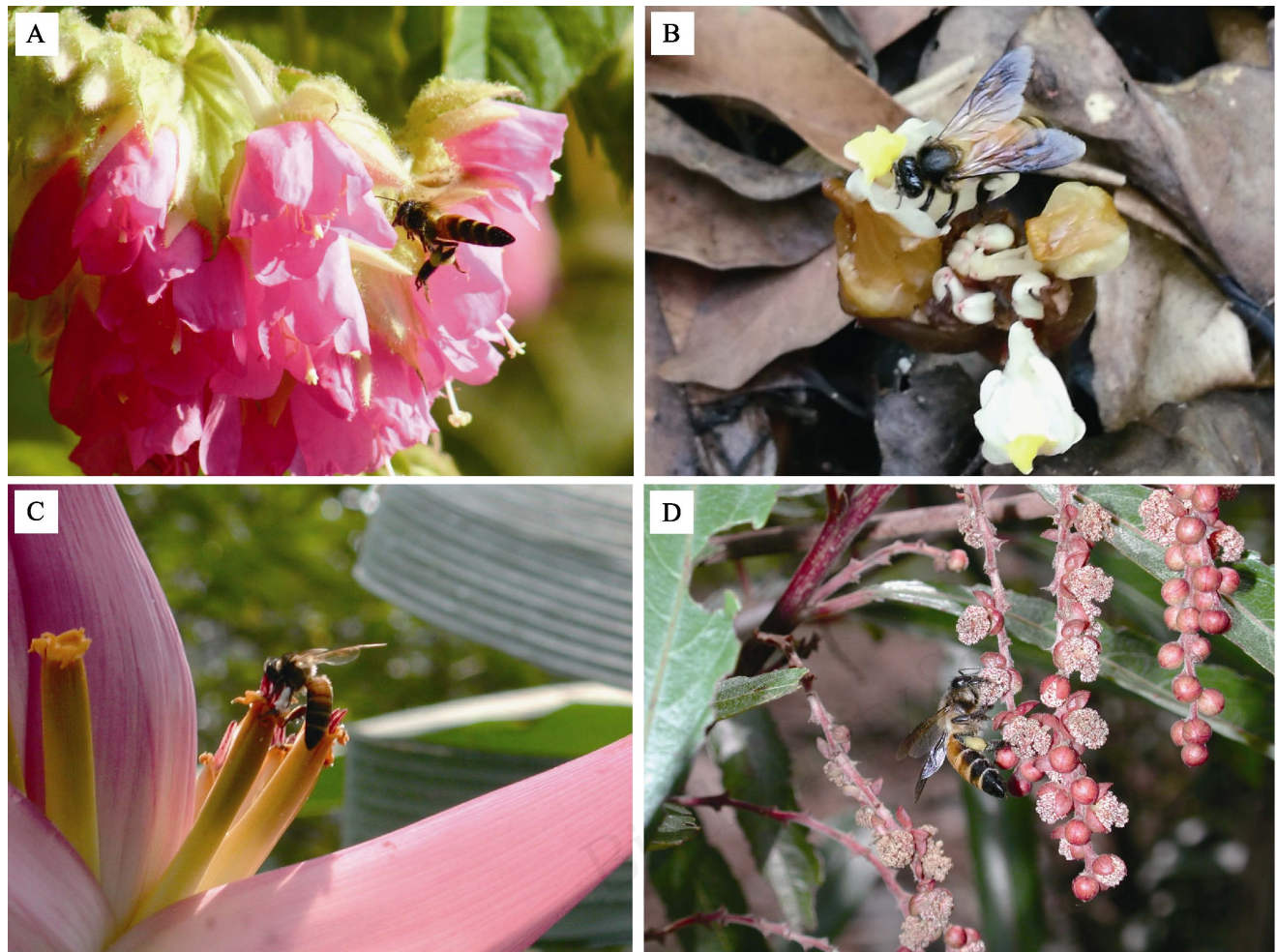

图3 大蜜蜂传粉的4种热带植物。A：非洲芙蓉; B：阳春砂仁; C: 紫苞芭蕉; D: 水柳。

Fig. 3 Apis dorsata visiting flowers of four tropical plants. A, Dombeya acutangula; B, Amomum villosum; C, Musa ornata; D, Homonoia riparia. 
部结构已形成特殊的镜像花(mirror-image flowers), 有左偏花柱和右偏花柱两种花型, 根据花柱与雄荵 的距离分为大雌雄异位型和小雌雄异位型两类, 其 中小雌雄异位型则完全依赖大蜜蜂传粉(Ren et al, 2013; 钱贞娜等, 2016)。当小雌雄异位型的风筝果 开花时，花柱与花药之间的间距恰好与大蜜蜂腹部 宽度相等, 大蜜蜂访问一种偏向花柱花型时, 腹部 一侧粘附花粉; 而当访问另一种花型时, 粘附花粉 的腹部与该花柱头接触完成传粉, 此时腹部另一侧 可与访花的花药接触, 借此避免同种花型自交, 实 现异花传粉(任明迅和张大勇, 2004)。实际上, 这反 映了在不同分布区风筝果与大蜜蜂之间的形态和 行为的适应性进化, 导致了种群间基因流分化, 从 而促进了物种形成, 也可进一步解释为何小雌雄异 位型风筝果仅生长在大蜜蜂的分布区的原因(钱贞 娜等, 2016)。

此外, 大蜜蜂还是众多热带农作物的主要和有 效传粉者(Gallai et al, 2009)。在印度、巴基斯坦和 泰国等地, Potts等(2003)发现有21科40种热带作物 需要依赖大蜜蜂传粉, 尤其是咖啡和芒果, 前者的 传粉昆虫中有 $56 \%$ 为大蜜蜂, 后者是大蜜蜂临时聚 集休整的重要场所和传粉植物 (Robinson, 2012; Boreux et al, 2013)。尽管家养的西方蜜蜂和东方蜜 蜂被认为是果蔬等农作物访花频率最高的有效传 粉者, 但一些地区大蜜蜂的访花时间和效率也很高, 有时甚至高于前两者(Ali et al, 2014; Dorjay et al, 2017; Shankar et al, 2017; Srivastava et al, 2017)。我 们在研究阳春砂仁(Amomum villosum)的传粉昆虫 时，发现大蜜蜂的采粉率达到 $56.99 \%$, 而家养东方 蜜蜂仅有 $3.37 \%$ (未发表数据)。由此可见, 大蜜蜂传 粉作用不仅实现了热带地区重要农作物的有性繁 殖, 促进基因交流, 而且还对农作物生产、食品安 全及人类福祉有着不可替代的作用。

\section{2 大蜜蜂种群数量面临的威胁}

\section{1 人为猎取蜂巢}

猎取蜂巢是一种直接破坏大蜜蜂栖息场所的 暴力式采蜜行为, 潜在危害巨大。一方面, 大蜜蜂 单个蜂巢容纳的个体量非常大，几百只到十万只不 等(Oldroyd et al, 2000), 采蜜人为采集蜂蜜和蜂蜡, 获得大蜜蜂的幼虫和蛹, 猎取整个巢穴, 破坏巢址, 致使迁飞回来的大蜜蜂难于重新建巢, 种群发展潜
能受阻(Lahjie \& Seibert, 1990; Soman \& Kshirsagar, 1991; Nath et al, 1994)。另一方面，浓烟或大火熏烧 蜂巢是目前常用的猎取手段, 若发生在白天, 蜂群 还可逃逸，重新选址建巢。但一些采蜜人为避免被 大蜜蜂蜇伤，常会选择在晚上熏巢或直接喷酒杀虫 剂, 造成大蜜蜂的成年个体(主要是工蜂), 有时甚 至是蜂王死亡, 严重侵扰了大蜜蜂种群的正常繁衍 和分巢能力(Lahjie \& Seibert, 1990; Nath et al, 1994; Oldroyd \& Nanork, 2009)。

\section{2 栖息生境遭受破坏}

栖息生境的组成和空间结构是物种种群数量 的关键驱动力(Neokosmidis et al, 2018)。由于近年来 城镇化进程加快、经济作物和经济林木大量推广种 植、原始森林砍伐等人为活动，区域内生境大小、 组成和结构均受到严重破坏，特别是东南亚的热带 雨林，预测本世纪将有 $42 \%$ 的物种多样性丧失 (Sodhi et al, 2004), 意味着适合大蜜蜂的筑巢树木 及其蜜粉源植物都极可能减少或消失，将增加大蜜 蜂发现合适栖息地和食物源的难度。同时，热带雨 林生境碎片化日益严重, 生境结构随之发生变化, 大蜜蜂可能不合时宜地被迫迁飞，无法与先前蜜粉 源植物物候期相吻合，极可能无法及时搜寻到合适 的蜜粉源，甚至在整个迁飞过程中都无法临时筑巢 休整，加之飞行环境的不适宜性，最终会导致大蜜 蜂个体死亡和种群数量的降低(Deutsch et al, 2008; Ollerton, 2017)。

\section{3 杀虫剂和除草剂滥用}

杀虫剂和除草剂是两类应用最为广泛的化学 农药，在调控有害生物、保障农林业正常生产方面 有着重要作用, 但也给生态环境带来了严重污染和 潜在危害(Kremen et al, 2007; Oldroyd \& Nanork, 2009; Sihag, 2014)。为了控制有害生物，荔枝园、芒 果园、龙眼园等热带果园常会喷酒杀虫剂和除草剂, 但这些药剂仅 $1 \%$ 起到了杀虫除草的目的，剩余 $99 \%$ 的药剂则进入了土壤、大气、水流，杀死传粉昆虫 等非靶标生物。像大蜜蜂这样的野生传粉昆虫, 对 化学农药反应尤为敏感, 忍耐力较低, 与杀虫剂和 除草剂接触即会致使其采蜜量减少、受食效率降低, 繁殖功能衰退甚至无法繁殖, 严重时个体直接死亡 (Colla, 2016)。此外，果农常常不按规定配置农药比 例，随意提高剂量、增加施药频率、使用广谱性药 剂等现象普遍，使得大蜜蜂长时间生活在高浓度农 
药的胁迫生境中, 大大增加了死亡概率。另一方面, 进入溪流的化学药剂势必会对在溪流或水源地附 近受食休整的大蜜蜂产生毒害, 间接消减了大蜜蜂 种群增长的潜能(Oldroyd \& Nanork, 2009)。

\section{4 昆虫、螨类和病原物侵染}

大蜜蜂会被一些昆虫和螨类寄生, 也会被真 菌、细菌、病毒等病原微生物感染, 甚至被两种或 多种病原微生物共同侵染, 引起并发症, 致使大蜜 蜂个体甚至整巢死亡(Laigo \& Morse, 1968; Oldroyd \& Nanork, 2009; Warrit \& Lekprayoon, 2011; Chantawannakul et al, 2016)。螨类是蜜蜂最为重要的外寄 生虫, 寄生大蜜蜂的主要是小蜂螨(Tropilaelaps spp.), 有亮热厉螨 ( $T$. clareae)、梅氏热厉螨 ( $T$. mercedesae)和柯氏热厉螨(T. koenigerum)三种，尤 以梅氏热厉螨威胁最大, 不仅直接引起大蜜蜂个体 死亡, 而且会引起蜂群蜂螨综合征 (parasitic mite syndrome, PMS), 并与蜜蜂病毒病、细菌病和真菌 病有着直接的关系(Laigo \& Morse, 1968; Sammataro et al, 2000; Warrit \& Lekprayoon, 2011; Chantawannakul et al, 2016)。另外，一些寄生性或捕食性昆 虫也是不可忽略的, 如双翅目眼蝇 (Physocephala parralleliventris)可将卵产在正飞行的大蜜蜂身体上, 卵孵化后即刻取食大蜜蜂体内的营养物质, 待其羽 化后, 大蜜蜂个体随即死亡(Rattanawannee \& Chanchao, 2011)。最具潜在威胁性的是南非小蜂窝甲虫 (Aethina tumida), 一旦该甲虫在蜜蜂蜂巢内定殖, 可在一天内攻击巢内所有幼虫, 致使整个蜂巢内的 蜜蜂逃离或者死亡(Ellis \& Hepburn, 2006), 且南非 小蜂窝甲虫偏爱温暖潮湿的气候, 入侵性极强, 目 前已侵入美国、澳大利亚和埃及等地(Ellis \& Hepburn, 2006)。因此, 南非小蜂窝甲虫对于生活在热 带雨林且密集建巢的大蜜蜂的潜在危害需要密切 关注。

\section{5 气候变化的影响}

在热带地区, 气候的微小改变即会对热带生物, 特别是数量众多的热带昆虫产生严重的影响, 因为 它们是无法适应或迁移的(Deutsch et al, 2008)。对于 大蜜蜂来说, 虽然目前没有相关研究显示气候变化 对其分布范围的影响, 但根据同属蜜蜂的研究, 气 候变化极可能造成大蜜蜂分布范围缩小(Potts et al, 2010; Ghazoul, 2015; Kerr et al, 2015)。此外, 由于南 半球气候相对长期稳定(Pauw \& Stanway, 2015), 热
带地区蜜蜂多样性相对较低, 南半球热带植物与访 花传粉昆虫之间的网络互作专化性呈增加的趋势 (Ollerton, 2017)。而北半球拥有丰富的生物多样性, 缺乏这种专化性趋势, 意味着气候变化容易改变生 境组成和空间结构, 引起蜜粉源植物花期变化, 从 而直接影响大蜜蜂筑巢、受食、传粉等生态学特征, 引起大蜜蜂种群的消减(Oldroyd \& Nanork，2009; Lebuhn et al, 2013)。

\section{3 大蜜蜂种质资源的保护与利用}

大蜜蜂是主要分布于亚洲热带的野生传粉昆 虫, 对该地区生态系统和生物多样性的维持和稳定 有着重要作用, 但随着威胁其种群数量的各种因素 增强，大蜜蜂的生存环境日益恶化(Nath et al, 1994; Ollerton, 2017; Srivastava et al, 2017; Neokosmidis et al, 2018)。因而, 有必要因地制宜地制定大蜜蜂种质 资源保护和可持续利用策略。

\section{1 强化大蜜蜂基础研究和保护工作}

与家养蜜蜂相比, 大蜜蜂基础研究明显滞后, 甚至对一些基础生物、生态学特征, 诸如生活习性、 繁殖特征、优良品系选育等知之甚少，无法为大蜜 蜂种质资源保护提供技术支撑。这就需要一方面强 化基础研究, 深入了解和掌握大蜜蜂种群遗传学、 养蜂学、进化生物学等知识, 用科学的方法进行人 工或半人工干预、驯化、选育优良品系。例如，在 越南南部, 养蜂人从 20 世纪末即在白千层(Melaleuca leucadendron)林开阔地用椽子吸引大蜜蜂前 来筑巢, 在所搭建的椽子中, 大蜜蜂筑巢率可达 $60 \%$, 而林中蜂巢数量也会相应增加，养蜂人总收 蜜量大幅度提高(Tan et al, 1997; Tan \& Ha, 2002)。 另一方面, 根据大蜜蜂生活环境的气候特点、植被 构成、蜜源植物种类和分布、大蜜蜂资源利用模式、 热带农作物耕作方式、种植结构变化规律等, 开展 与传粉植物互作网络关系与评价的研究, 改善生态 环境，建立分布合理的蜜源植物基地，保障种群发 展潜能(Robinson, 2012; Ollerton, 2017)。

\section{2 推动生态农业和其他产业融合发展}

从生态农业整体功能出发, 按照“协调、循环、 再生”的原则调整和优化热带农林产业的整体结构, 融合其他相关产业，提高综合生产力和经济价值。 首先，针对杀虫剂、除草剂、除螨剂、除菌剂等滥 用的问题，建议减少使用这些化学药剂，尽量改用 
低毒、无毒、低残留的生物源制剂, 并与灯诱、粘 板、性诱剂、聚集素等绿色控虫技术相结合(Oldroyd \& Nanork, 2009; Colla, 2016), 最大程度降低对大蜜 蜂的直接毒害, 保护蜜源健康, 为大蜜蜂种群繁衍 营造良好的栖息环境。其次, 开发大蜜蜂及其蜂产 品旅游项目, 将景观农业与旅游和科普产业融合在 一起, 正如马来西亚和尼泊尔地区, 已有大量游 客、养蜜人和蜜蜂爱好者参观几十米高的大树或岩 壁上的大蜜蜂巢, 听取大蜜蜂科普趣闻, 购买相关 产品。这是一种多赢措施, 不仅有利于提高居民的 旅游收入, 更可带动周边相关产业, 弘扬蜜蜂文化 知识, 提高人们对大蜜蜂的科学认知, 促进人与动 物的和谐共存, 从而更好地保护大蜜蜂种质资源。 最后, 大蜜蜂保护可与传粉业进行整合, 特别是当 今, 规模化种植和设施农业迅猛发展, 热带作物集 中开花时急需大量传粉昆虫, 而大蜜蜂又具有传粉 效率高、采蜜量大等优点, 有利于大蜜蜂传粉产 业的发展, 进而实现大蜜蜂种质资源保护与传粉 业共赢。

\section{3 建立适合大蜜蜂迁飞的生态廊道}

目前, 尽管尚不清楚热带雨林消失和生境碎片 化对大蜜蜂种质资源的影响程度, 但生境丧失和蜜 粉源植物减少势必对大蜜蜂筑巢地、迁飞路径、迁 飞目的地、临时休整等有负面影响(Kevan \& Viana, 2003; Sodhi et al, 2004)。因而, 可以在大蜜蜂的迁飞 路线上建立热带雨林自然保护区, 或者种植大蜜蜂 喜爱的蜜粉源植物(Crane et al, 1993; Oldroyd \& Nanork, 2009), 如芒果树, 大蜜蜂既可以采粉又可 筑巢, 形成有利于大蜜蜂迁飞休整的生态廊道。在 城市化的过程中, 尤其在大蜜蜂分布核心区, 选择 适合大蜜蜂筑巢的高大乔木, 如木棉和高榕(Ficus altissima), 或偏好的蜜粉源植物, 构建城市绿地、 公园、植物园等多层次景观, 保证大蜜蜂在局域空 间内能够筑巢和发现食物资源(Noreen et al, 2016)。 同时, 加强对这些区域内大蜜蜂种群数量的动态监 测, 评估生态廊道和城市绿地对大蜜蜂种质资源 的影响程度, 促进大蜜蜂种群数量增长和栖息地 改善。

\section{4 加强检验检疫}

随着技术的进步和农林生产的快速发展, 国际 贸易和人员流动日益频繁, 无形之中增大了有害生 物的传入概率, 特别是对大蜜蜂有致命性质的, 如
小蜂螨和南非小蜂窝甲虫, 严重影响大蜜蜂种群的 繁衍(Ellis \& Hepburn, 2006)。因而, 要尽可能防止 这些有害生物的入侵，但目前仍不清楚寄生或侵染 大蜜蜂的昆虫、螨及病原物的种类、传播途径及其 潜在危害(Chantawannakul et al, 2016)，这限制了检 验检疫措施的实施，但我们可以参考家养蜜蜂、熊 蜂、或其他野生蜜蜂等相关案例。例如, 马来西亚 严禁西方蜜蜂输入婆罗洲, 韩国和日本禁止从有南 非小蜂窝甲虫分布的国家或地区进口蜂王和蜂箱 (Oldroyd \& Nanork, 2009)。与此相应, 对养蜂人的 蜜蜂也需加强抽查和监控力度, 一旦发现有寄生或 病原物感染, 特别是出现诸如蜂巢口有大量爬蜂、 蜂群规模逐渐减少、幼虫腐臭等症状, 应立即采取 扣王断子法、雄蜂脾诱杀法、烟草烟雾熏杀等脱毒 减毒措施(Woyke, 1984; Sammataro et al, 2000), 限 制这些蜜蜂的输出。最为重要的是, 加强对大蜜蜂 有侵染性的昆虫、螨、病原物等的基础研究, 为 开展大蜜蜂检验检疫和保护其种质资源提供科学 依据。

\section{5 科学合理利用大蜜蜂种质资源}

当前, 人为暴力式猎取蜂巢是大蜜蜂种群数量 减少的主要因素。国外通过长期调查和监测大蜜蜂 的寿命、交尾、繁殖、分巢、迁飞等生物学特性，来 综合评估大蜜蜂资源的可利用性，计算单位面积单 位时间内可收割的大蜜蜂蜂巢数量，在一定范围一 定时间内进行配额采割。然而, 天然野生资源量毕 竟有限, 无法满足人们对绿色生态食品的强烈渴求, 使得蜂产品价格鄚升, 刺激采蜜人大肆掠夺蜂巢, 这就需要将大蜜蜂种群保护与其他产业融合发展, 综合开发(Tan \& Ha, 2002)。一般来说, 大蜜蜂多栖 于生态环境良好的原始森林内, 在高大乔木枝干上 建巢，而这恰好符合当今人们渴望回归自然和探求 刺激有趣的生活，与旅游业结合有其得天独厚的优 势, 马来西亚旅游胜地金马屯开发的大蜜蜂蜂巢割 取参观即是一个很好的案例，取得了良好的社会、 经济和生态效益。相比之下, 我国大蜜蜂分布范围 窄，仅在云南、广西和海南热带地区有分布，也见 人为暴力式猎取、贩卖大蜜蜂蜂巢和蜂蜜的现象, 在没有很好保护和利用的背景下, 呼吁禁止人为猎 取、破坏大蜜蜂蜂巢的行为, 保护这一热带重要的 传粉昆虫资源。对于热带种植业，大蜜蜂的适时访 花授粉也是必不可少的, 有利于提高作物结果率和 
改善果实品质，大幅度降低落果率和畸果率，提高 粮食生产和食品安全, 推动产业结构调整和可持续 发展。可以预见，未来大蜜蜂种质资源的科学合理 利用，必须与旅游业、授粉业、种植业、自然保护 区规划等相结合，方可解决种质保护与开发利用的 核心冲突问题，实现大蜜蜂资源的永续利用。

\section{参考文献}

Alexandra-Maria K, Bernard EV, James HC, Ingolf S-D, Saul AC, Claire K, Teja T (2007) Importance of pollinators in changing landscapes for world crops. Proceedings of the Royal Society B, 274, 303-313.

Ali M, Saeed S, Sajjad A, Bashir MA (2014) Exploring the best native pollinators for pumpkin (Cucurbita pepo) production in Punjab, Pakistan. Pakistan Journal of Zoology, 46, 531-539.

Boreux V, Krishnan S, Cheppudira KG, Ghazoul J (2013) Impact of forest fragments on bee visits and fruit set in rain-fed and irrigated coffee agro-forests. Agriculture, Ecosystems \& Environment, 172, 42-48.

Breeze TD, Vaissière BE, Bommarco R, Petanidou T, Seraphides N, Kozák L, Scheper J, Biesmeijer JC, Kleijn D, Gyldenkærne S (2014) Agricultural policies exacerbate honeybee pollination service supply-demand mismatches across Europe. PLoS ONE, 9, e82996.

Burkle LA, Marlin JC, Knight TM (2013) Plant-pollinator interactions over 120 years: Loss of species, co-occurrence, and function. Science, 339, 1611-1615.

Cameron SA, Lozier JD, Strange JP, Koch JB, Cordes N, Solter LF, Griswold TL (2011) Patterns of widespread decline in North American bumble bees. Proceedings of the National Academy of Sciences, USA, 108, 662-667.

Cao LF, Hu FL (2012) Biological characters of Apis dorsata from China. Journal of Bee, (4), 1-2. (in Chinese) [曹联飞, 胡福良 (2012) 中国大蜜蜂生物学特性研究初报. 蜜蜂 杂志, (4), 1-2.]

Chantawannakul P, de Guzman LI, Li J, Williams GR (2016) Parasites, pathogens, and pests of honeybees in Asia. Apidologie, 47, 301-324.

Colla SR (2016) Status, threats and conservation recommendations for wild bumble bees (Bombus spp.) in Ontario, Canada: A review for policymakers and practitioners. Natural Areas Journal, 36, 412-426.

Crane E, van Luyen V, Mulder V, Ta TC (1993) Traditional management system for Apis dorsata in submerged forests in southern Vietnam and central Kalimantan. Bee World, 74, 27-40.

Deutsch CA, Tewksbury JJ, Huey RB, Sheldon KS, Ghalambor CK, Haak DC, Martin PR (2008) Impacts of climate warming on terrestrial ectotherms across latitude. Proceedings of the National Academy of Sciences, USA, 105, 6668-6672.
Dorjay N, Abrol DP, Shankar U (2017) Insect visitors on cucumber and bittergourd flowers and impact on quantity of crop production by different pollination treatment. Journal of Apiculture, 32, 77-88.

Dyer FC, Seeley T (1994) Colony migration in the tropical honey bee Apis dorsata F. (Hymenoptera: Apidae). Insectes Sociaux, 41, 129-140.

Dyer FC (1985) Nocturnal orientation by the Asian honey bee, Apis dorsata. Animal Behaviour, 33, 769-774.

Ellis J, Hepburn H (2006) An ecological digest of the small hive beetle (Aethina tumida), a symbiont in honey bee colonies (Apis mellifera). Insectes Sociaux, 53, 8-19.

Gallai N, Salles J-M, Settele J, Vaissière BE (2009) Economic valuation of the vulnerability of world agriculture confronted with pollinator decline. Ecological Economics, 86, 810-821.

Ghazoul J (2015) Qualifying pollinator decline evidence. Science, 348, 981-982.

Hepburn H, Radloff SE (2011) Biogeography. In: Honeybees of Asia (eds Hepburn R, Radloff S), pp. 51-67. Springer, Heidelberg.

Itioka $\mathrm{T}$, Inoue $\mathrm{T}$, Kaliang $\mathrm{H}$, Kato $\mathrm{M}$, Nagamitsu $\mathrm{T}$, Momose K, Sakai S, Yumoto T, Mohamad SU, Hamid AA (2001) Six-year population fluctuation of the giant honey bee Apis dorsata (Hymenoptera: Apidae) in a tropical lowland dipterocarp forest in Sarawak. Annals of the Entomological Society of America, 94, 545-549.

Kerr JT, Pindar A, Galpern P, Packer L, Potts SG, Roberts SM, Rasmont P, Schweiger O, Colla SR, Richardson LL (2015) Climate change impacts on bumblebees converge across continents. Science, 349, 177-180.

Kevan PG, Viana BF (2003) The global decline of pollination services. Biodiversity, 4, 3-8.

Kremen C, Williams NM, Aizen MA, Gemmill-Herren B, LeBuhn G, Minckley R, Packer L, Potts SG, Steffan-Dewenter I, Vazquez DP (2007) Pollination and other ecosystem services produced by mobile organisms: A conceptual framework for the effects of land-use change. Ecology Letters, 10, 299-314.

Lahjie AM, Seibert B (1990) Honey gathering by people in the interior of East Kalimantan. Bee World, 71, 153-157.

Laigo FM, Morse RA (1968) The mite Tropilaelaps clareae in Apis dorsata colonies in the Philippines. Bee World, 49, 116-118.

Lebuhn G, Droege S, Connor EF, Gemmill-Herren B, Potts SG, Minckley RL, Griswold T, Jean R, Kula E, Roubik DW (2013) Detecting insect pollinator declines on regional and global scales. Conservation Biology, 27, 113-120.

Makinson JC, Schaerf TM, Rattanawannee A, Oldroyd BP, Beekman M (2014) Consensus building in giant Asian honeybee, Apis dorsata, swarms on the move. Animal Behaviour, 93, 191-199.

Momose K, Yumoto T, Nagamitsu T, Kato M, Nagamasu H, 
Sakai S, Harrison RD, Itioka T, Hamid AA, Inoue T (1998) Pollination biology in a lowland dipterocarp forest in Sarawak, Malaysia. I. Characteristics of the plant-pollinator community in a lowland dipterocarp forest. American Journal of Botany, 85, 1477-1501.

Moreyra S, D’Adamo P, Lozada M (2017) Long-term spatial memory in Vespula germanica social wasps: The influence of past experience on foraging behavior. Insect Science, 24, 853-858.

Nagir MT, Atmowidi T, Kahono S (2016) The distribution and nest-site preference of Apis dorsata Binghami at Maros forest, South Sulawesi, Indonesia. Journal of Insect Biodiversity, 4, 1-14.

Nath S, Roy P, Leo R, John M (1994) Honeyhunters and Beekeepers of Tamil Nadu, A Survey Document. Coonoor Printing Press, Tamil Nadu.

Neokosmidis L, Tscheulin T, Devalez J, Petanidou T (2018) Landscape spatial configuration is a key driver of wild bee demographics. Insect Science, 25, 172-182.

Neumann P, Koeniger N, Koeniger G, Tingek S, Kryger P, Moritz RF (2000) Home-site fidelity in migratory honeybees. Nature, 406, 474-475.

Noreen A, Niissalo M, Lum S, Webb E (2016) Persistence of long-distance, insect-mediated pollen movement for a tropical canopy tree species in remnant forest patches in an urban landscape. Heredity, 117, 472-480.

Oldroyd B, Osborne K, Mardan M (2000) Colony relatedness in aggregations of Apis dorsata Fabricius (Hymenoptera, Apidae). Insectes Sociaux, 47, 94-95.

Oldroyd BP, Nanork P (2009) Conservation of Asian honey bees. Apidologie, 40, 296-312.

Ollerton J (2017) Pollinator diversity: Distribution, ecological function, and conservation. Annual Review of Ecology, Evolution, and Systematics, 48, 353-376.

Ollerton J, Erenler H, Edwards M, Crockett R (2014) Extinctions of aculeate pollinators in Britain and the role of large-scale agricultural changes. Science, 346, 1360-1362.

Paar J, Oldroyd B, Huettinger E, Kastberger G (2004) Genetic structure of an Apis dorsata population: The significance of migration and colony aggregation. Journal of Heredity, 95, 119-126.

Paar J, Oldroyd B, Kastberger G (2000) Giant honeybees return to their nest sites. Nature, 406, 475.

Pauw A (2007) Collapse of a pollination web in small conservation areas. Ecology, 88, 1759-1769.

Pauw A, Stanway R (2015) Unrivalled specialization in a pollination network from South Africa reveals that specialization increases with latitude only in the Southern Hemisphere. Journal of Biogeography, 42, 652-661.

Potts SG, Biesmeijer JC, Kremen C, Neumann P, Schweiger O, Kunin WE (2010) Global pollinator declines: Trends, impacts and drivers. Trends in Ecology \& Evolution, 25, 345-353.
Potts SG, Vulliamy B, Dafni A, Ne’eman G, Willmer P (2003) Linking bees and flowers: How do floral communities structure pollinator communities? Ecology, 84, 2628-2642.

Potts SG, Imperatriz-Fonseca VL, Ngo HT, Biesmeijer JC, Breeze TD, Dicks LV, Garibaldi LA, Hill R, Settele J, Vanbergen AJ, Aizen MA, Cunningham SA, Eardley C, Freitas BM, Gallai N, Kevan PF, Kovács-Hostyánszki A, Kwapong PK, Li J, Li X, Martins DJ, Nates-Parra G, Pettis JS, Rader R, Viana BF (2016) IPBES: Summary for Policymakers of the Assessment Report of the Intergovernmental Science-Policy Platform on Biodiversity and Ecosystem Services on Pollinators, Pollination and Food Production. Secretariat of the Intergovernmental Science-Policy Platform on Biodiversity and Ecosystem Services, Bonn.

Qian ZN, Meng QW, Ren MX (2016) Pollination ecotypes and herkogamy variation of Hiptage benghalensis (Malpighiaceae) with mirror-image flowers. Biodiversity Science, 24, 1364-1372. (in Chinese with English abstract) [钱贞娜, 孟 千万, 任明迅 (2016) 风筝果镜像花的雌雄异位变化及 传粉生态型的形成. 生物多样性, 24, 1364-1372.]

Raghunandan K, Basavarajappa S (2014) Floral hosts and pollen calendar of Asian giant honeybee, Apis dorsata Fabricius at Southern Karnataka, India. Journal of Ecology and the Natural Environment, 6, 321-330.

Rattanawannee A, Chanchao C (2011) Bee diversity in Thailand and the applications of bee products. In: Changing Diversity in Changing Environment (eds Grillo O, Venora G), pp. 152-154. InTech, Rijeka.

Rattanawannee A, Chanchao C, Lim J, Wongsiri S, Oldroyd BP (2013) Genetic structure of a giant honey bee (Apis dorsata) population in northern Thailand: Implications for conservation. Insect Conservation and Diversity, 6, 38-44.

Ren MX, Zhang DY (2004) Herkogamy. In: Plant Life-History Evolution and Reproductive Ecology (ed. Zhang DY), pp. 302-321. Science Press, Beijing. (in Chinese) [任明迅, 张 大勇 (2004) 䧳雄异位. 见: 植物生活史进化与繁殖生态 学 (张大勇主编), 302-321页. 科学出版社, 北京.]

Ren MX, Zhong YF, Song XQ (2013) Mirror-image flowers without buzz pollination in the Asian endemic Hiptage benghalensis (Malpighiaceae). Botanical Journal of the Linnean Society, 173, 764-774.

Robinson WS (2012) Migrating giant honey bees (Apis dorsata) congregate annually at stopover site in Thailand. PLoS ONE, 7, e44976.

Sammataro D, Gerson U, Needham G (2000) Parasitic mites of honey bees: Life history, implications, and impact. Annual Review of Entomology, 45, 519-548.

Shankar U, Abrol D, Chatterjee D, Rizvi S (2017) Diversity of native bees on Parkinsonia aculeata L. in Jammu region of North-West Himalaya. Tropical Ecology, 58, 211-215.

Sihag RC (2014) Phenology of migration and decline in colony numbers and crop hosts of giant honeybee (Apis dorsata F.) in semiarid environment of Northwest India. Journal of 
Insects, 2014, 1-9.

Sihag RC (2017) Nesting behavior and nest site preferences of the giant honey bee (Apis dorsata F.) in the semi-arid environment of north west India. Journal of Apicultural Research, 56, 452-466.

Sodhi NS, Koh LP, Brook BW, Ng PK (2004) Southeast Asian biodiversity: An impending disaster. Trends in Ecology \& Evolution, 19, 654-660.

Soman A, Kshirsagar K (1991) Preliminary survey on the rockbee (Apis dorsata F.) and some observations on the traditional methods of honey hunting. Indian Bee Journal, 53, 17-22.

Somanathan H, Warrant EJ, Borges RM, Wallén R, Kelber A (2009) Resolution and sensitivity of the eyes of the Asian honeybees Apis florea, Apis cerana and Apis dorsata. Journal of Experimental Biology, 212, 2448-2453.

Srivastava K, Sharma D, Pandey S, Anal A, Nath V (2017) Dynamics of climate and pollinator species influencing litchi (Litchi chinensis) in India. Indian Journal of Agricultural Sciences, 87, 266-269.

Starr CK, Schmidt PJ, Schmidt JO (1987) Nest-site preferences of the giant honey bee, Apis dorsata (Hymenoptera: Apidae), in Borneo. Pan-Pacific Entomologist, 63, 37-42.

Tan NQ, Chinh PH, Thai PH, Mulder V (1997) Rafter beekeeping with Apis dorsata: Some factors affecting the occupation of rafters by bees. Journal of Apicultural Research,
36, 49-54.

Tan NQ, Ha DT (2002) Socio-economic factors in traditional rafter beekeeping with Apis dorsata in Vietnam. Bee World, 83, 165-170.

Wardhaugh CW (2015) How many species of arthropods visit flowers? Arthropod-Plant Interactions, 9, 547-565.

Warrit N, Lekprayoon C (2011) Asian honeybee mites. In: Honeybees of Asia (eds Hepburn R, Radloff S), pp. 347-368. Springer, Heidelberg.

Woyke J (1984) Survival and prophylactic control of Tropilaelaps clareae infesting Apis mellifera colonies in Afghanistan. Apidologie, 15, 421-434.

Woyke J, Wilde J, Wilde M (2012) Swarming and migration of Apis dorsata and Apis laboriosa honey bees in India, Nepal and Bhutan. Journal of Apicultural Science, 56, 81-91.

Wu YR (2000) Fauna Sinica (Tomus 20), Insecta, Melittidae, Apidae. Science Press, Beijing. (in Chinese) [吴燕如 (2000) 中国动物志 (第20卷) 昆虫纲・准蜂科・蜜蜂科. 科学出版社, 北京.]

Zhang ZS (1974) Primary research on Apis dorsata Fabricius in Yunnan. Entomological Knowledge, (3), 42-44. (in Chinese) [张正松 (1974) 云南排蜂的初步研究. 昆虫知识, (3), 42-44.]

(责任编委: 黄双全 责任编辑: 问文杰) 